\title{
Multiplexing lobster-eye optics: a concept for wide-field $x$-ray monitoring
}

\author{
Toru Tamagawa, ${ }^{a, b, c, *}$ Keisuke Uchiyama, ${ }^{\text {b,c }}$ Ryota Otsubo, ${ }^{d}$ \\ Tatsuya Yuasa, ${ }^{d}$ Yuanhui Zhou, ${ }^{b, c}$ Tatehiro Mihara, ${ }^{\text {a,b }}$ Yuichiro Ezoe, ${ }^{d}$ \\ Masaki Numazawa, ${ }^{d}$ Daiki Ishi, ${ }^{d}$ Aoto Fukushima, ${ }^{d}$ Hikaru Suzuki, ${ }^{d}$ \\ Tomoki Uchino, ${ }^{d}$ Sae Sakuta, ${ }^{d}$ Kumi Ishikawa, ${ }^{\mathrm{e}}$ Teruaki Enoto, ${ }^{\mathrm{a}}$ and \\ Takanori Sakamoto ${ }^{f}$ \\ ${ }^{a}$ RIKEN Cluster for Pioneering Research, Wako, Saitama, Japan \\ ${ }^{b}$ RIKEN Nishina Center, Wako, Saitama, Japan \\ ${ }^{\mathrm{c}}$ Tokyo University of Science, Shinjuku, Tokyo, Japan \\ ${ }^{\mathrm{d}}$ Tokyo Metropolitan University, Hachioji, Tokyo, Japan \\ ${ }^{\mathrm{e}}$ Japan Aerospace Exploration Agency, Institute of Space and Astronautical Science, \\ Sagamihara, Kanagawa, Japan \\ ${ }_{\mathrm{f}}^{\mathrm{A}}$ oyama Gakuin University, Sagamihara, Kanagawa, Japan
}

\begin{abstract}
We propose a concept of multiplexing lobster-eye (MuLE) optics to achieve significant reductions in the number of focal plane imagers in lobster-eye (LE) wide-field x-ray monitors. In the MuLE configuration, an LE mirror is divided into several segments and the x-rays reflected on each of these segments are focused on a single image sensor in a multiplexed configuration. If each LE segment assumes a different rotation angle, the azimuthal rotation angle of a cross-like image reconstructed from a point source by the LE optics identifies the specific segment that focuses the x-rays on the imager. With a focal length of $30 \mathrm{~cm}$ and LE segments with areas of $10 \times 10 \mathrm{~cm}^{2}, \sim 1 \mathrm{sr}$ of the sky can be covered with $36 \mathrm{LE}$ segments and only four imagers (with total areas of $10 \times 10 \mathrm{~cm}^{2}$ ). A ray tracing simulation was performed to evaluate the nine-segment MuLE configuration. The simulation showed that the flux $(0.5$ to $2 \mathrm{keV})$ associated with the $5 \sigma$ detection limit was $\sim 2 \times 10^{-10} \mathrm{erg} \mathrm{cm}^{-2} \mathrm{~s}^{-1}(10 \mathrm{mCrab})$ for a transient with a duration of $100 \mathrm{~s}$. The simulation also showed that the direction of the transient for flux in the range of 14 to $17 \mathrm{mCrab}$ at $0.6 \mathrm{keV}$ was determined correctly with a $99.7 \%$ confidence limit. We conclude that the MuLE configuration can become an effective on-board device for small satellites for future $\mathrm{x}$-ray wide-field transient monitoring. (c) The Authors. Published by SPIE under a Creative Commons Attribution 4.0 Unported License. Distribution or reproduction of this work in whole or in part requires full attribution of the original publication, including its DOI. [DOI: 10.1117/1.JATIS.6.2 $.025003]$
\end{abstract}

Keywords: x-ray all-sky monitoring; transient monitor; lobster-eye optics; multiplexing lobstereye; small satellite.

Paper 20020 received Feb. 13, 2020; accepted for publication Jun. 16, 2020; published online Jun. 30, 2020.

\section{Introduction}

Wide-field x-ray monitors have been proven to be indispensable devices in time-domain astronomy in recent years. The precise and immediate localization of transient phenomena is critical for the revelation of their origin. For example, quick localization of gamma-ray bursts (GRBs) has revealed the origin of GRBs with long durations as collapsers. ${ }^{1}$ Gravitational waves from a neutron star merger were detected ${ }^{2}$ in 2017 and demarcated the onset of multimessenger astronomy. ${ }^{3}$ In 2018 , follow-up observations were carried out for the neutrino burst detected by IceCube, and the origin of this event was localized to an active galactic nucleus. ${ }^{4}$ In 2021 , the large synoptic survey telescope ${ }^{5}$ will begin its observations and will generate several million alerts per night. ${ }^{6}$ Identifying the high-energy counterparts of these visible transients is important

*Address all correspondence to Toru Tamagawa, E-mail: tamagawa@riken.jp 
for elucidating their origins. Correspondingly, in multimessenger astronomy, the use of a device that constantly monitors the universe with a wide field-of-view $(\mathrm{FoV})$ in the $\mathrm{x}$-ray energy band is essential.

Coded masks are used for wide FoV missions such as INTEGRAL, ${ }^{7}$ Swift/BAT, ${ }^{8}$ HETE/ $\mathrm{WXM},{ }^{9}$ and BeppoSAX/WFC, ${ }^{10}$ but in principle it is difficult to increase the sensitivity because of the interference caused by the diffuse cosmic x-ray background (CXB). The all-sky monitors on-board $\mathrm{RXTE}^{11}$ and MAXI ${ }^{12}$ improved the detection sensitivities by narrowing the FoV with pinhole camera or slit techniques and yielded excellent performance in the observation of faint x-ray sources. To compensate for the improved sensitivity, the sky coverage of a moment was restricted to a few $\%$ of the entire sky.

Lobster-eye (LE) optics ${ }^{13}$ represents the best possible observation equipment for missions that require a wide FoV and increased sensitivity. The LE optics reduces the influence of the CXB by focusing and concurrently securing a broad FoV. Several x-ray astronomical satellite missions, such as Einstein Probe,${ }^{14}$ ISS-Lobster, ${ }^{15}$ and HiZ-GUNDAM,${ }^{16}$ employ the LE optics. A disadvantage of the LE optics is the necessity for large-sized imagers at the focal plane. For example, the all-sky monitor mission LOBSTER requires a detector area spanning $5000 \mathrm{~cm}^{2}$ to cover $\sim 1 / 4$ of the entire sky. ${ }^{17}$ In this study, we describe the design, feasibility, and performance evaluation of a newly proposed idea of LE optics to reduce the number of imagers.

\section{Concept of Reduction of Focal Plane Imagers}

The LE mirror consists of many square, hollow cells that operate as X-ray reflectors tiled on a curved sphere with a radius $R$, as shown in Fig. 1. X-rays that originate from a point source are reflected twice on the adjacent walls of a square hollow cell [Fig. 1(b)] and are focused on a point on the focal plane with a radius of $R / 2$. When the incident angle of the $\mathrm{x}$-rays is different, the $\mathrm{x}$-rays are focused on another location on the focal plane. In combination with the image sensors placed at the focal plane, the LE optics realizes $\mathrm{x}$-ray imaging with a wide FoV that is not achievable with any other standard x-ray mirror optics. As shown in Fig. 1(b), since the X-rays reflected only once in the $X(Y)$ surface of a cell are focused in the $X_{\text {det }}\left(Y_{\text {det }}\right)$ direction but not in the $Y_{\text {det }}$ $\left(X_{\text {det }}\right)$ direction, the focus should be a line along $Y_{\text {det }}\left(X_{\text {det }}\right)$. Thus, those photons are focused on

(a)

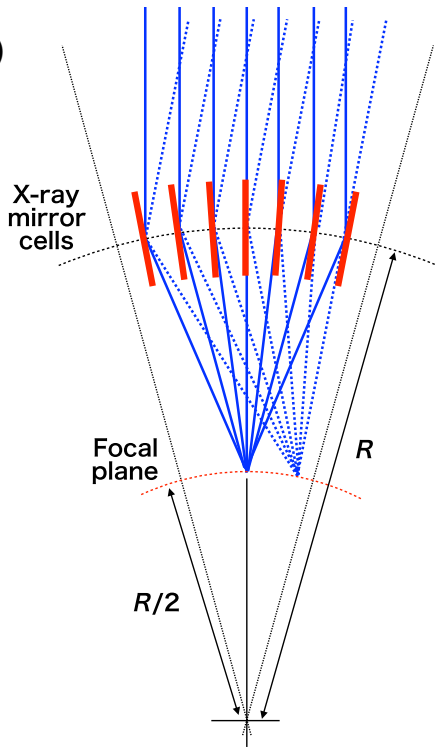

(b)

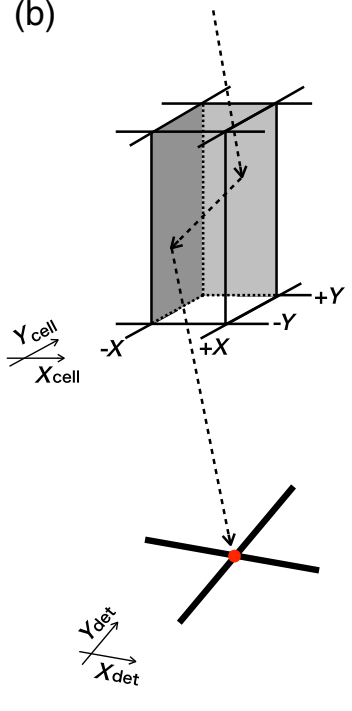

Fig. 1 (a) Schematics of the LE optics showing the $x$-ray mirror cells mounted on a curved spherical surface with a radius $R$ and focal plane detectors at a focal length of $R / 2$. X-rays from different positions in the sky are focused on different locations on the focal plane. (b) A square, hollow cell and the path of an $\mathrm{x}$-ray scattered on different planes $+Y$ and $-X$. The $\mathrm{x}$-ray photon is focused on the center of a cross-like image generated by the LE optics on a focal plane detector. The photons reflected on the mirror once are focused on the cross-like arm foci. 
cross-like arm foci. To cover the entire FoV of the LE mirror, which is the opening angle of an LE mirror segment as described in Appendix A, large image sensors covering a 1/4 size of the area of the LE mirror are required at the focal plane. However, imagers with large areas are sometimes unsuitable for a small satellite mission because they consume non-negligible satellite resources, such as electrical power, computer power, and data downlink bandwidth, and may cause cooling problems.

To overcome these disadvantages, we propose a new configuration in which the LE mirror is divided into several segments and the $\mathrm{x}$-rays reflected on each segment of the mirror are focused on a single, small image sensor, as shown in Fig. 2. If we define the opening angle of an LE segment as $2 \theta$, the LE segment ID20 in Fig. 2, which is $4 \theta$ away from the central segment (ID00), can be moved right next to the ID00 segment. We refer to this configuration in this study as "multiplexing lobster-eye (MuLE)" optics. To specify an LE segment, we use the notation ID $n_{\mathrm{x}} n_{\mathrm{y}}$, where $n_{\mathrm{x}}$ and $n_{\mathrm{y}}$ are indices used to represent the distance of the segment $2 n \theta$ away from ID00 in the $x$ and $y$ directions, respectively. Negative integers are represented with a bar. For example, $\bar{n}$ denotes $-n$. The similar configuration was adopted by the ABRIXAS mission ${ }^{18}$ in which one CCD camera was shared by seven X-ray mirrors. Their design was to drop different FoVs to different areas of the imager, but in our concept different FoVs are dropped to the same area of an imager.

How can we distinguish two stellar objects focused by different LE segments on one imager? As shown in Fig. 1(b), a point source focused by an LE segment shows a cross-like response on the imager. The azimuthal rotation angle of the cross-like arm foci on the imager is exactly the same as that of the square hollow cells of the LE segment around the central optical axis of the segment. By giving each segment a different azimuthal rotation angle, point sources focused by different mirror segments form cross-like arm foci with different azimuthal rotation angles.

We estimated the total FoV covered by the MuLE optics. The half angle $\theta$ of the FoV of each LE segment was defined as $\theta=\sin ^{-1}(L / 2 R)$, where each LE segment had an area of $L \times L$. If we consider specific values $R=60 \mathrm{~cm}$ and $L=10 \mathrm{~cm}$, the FoV of each segment becomes $9.6 \mathrm{deg} \times 9.6 \mathrm{deg}$. One of the possible configurations of the MuLE optics consists of nine tiled segments, as shown in Fig. 3, in which an azimuthal rotation angle of each LE segment increments $10 \mathrm{deg}$ from $0 \mathrm{deg}$ to $80 \mathrm{deg}$. The numbers $\phi 00-\phi 80$ in Fig. 3(a) indicate the azimuthal rotation angles of the LE segment cells around the optical axis. It is not difficult to manufacture such mirrors with current technology.

As observed from Fig. 2, the FoV covered by ID20 is not the continuous tiling of ID00 but a tiling configuration at every other position in the sky coordinate system. When four units of the

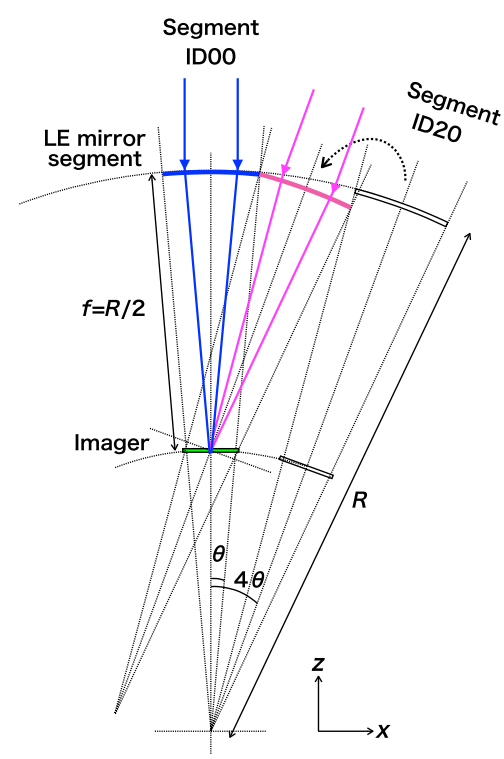

Fig. 2 Conceptual design of the MuLE optics to achieve reductions in the number of imagers. A single imager is shared between ID00 and ID20 LE mirror segments. 
(a)

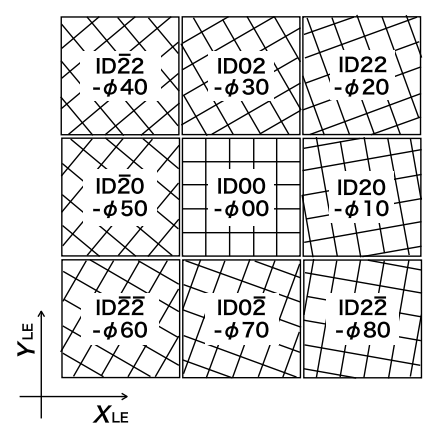

(b)

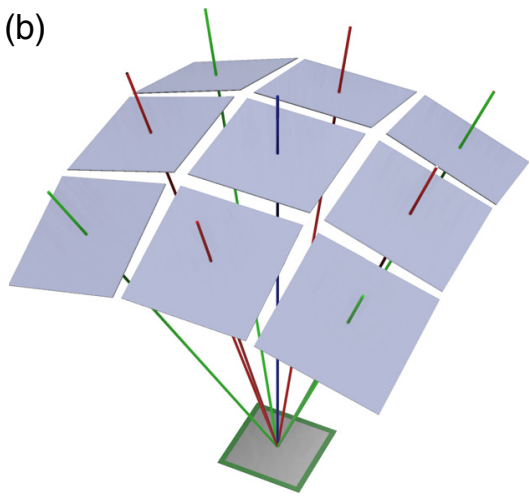

Fig. 3 (a) The configuration of nine-segment MuLE optics. The azimuthal rotation angle of the square, hollow cells of each segment is shifted by $10 \mathrm{deg}$. The numbers begin with $\phi$ after the use of the LE segment ID to represent the azimuthal rotation angles around the optical axes of the LE segments. The cell size of each segment is exaggerated. (b) Three-dimensional modeling of the nine-segment MuLE optics.

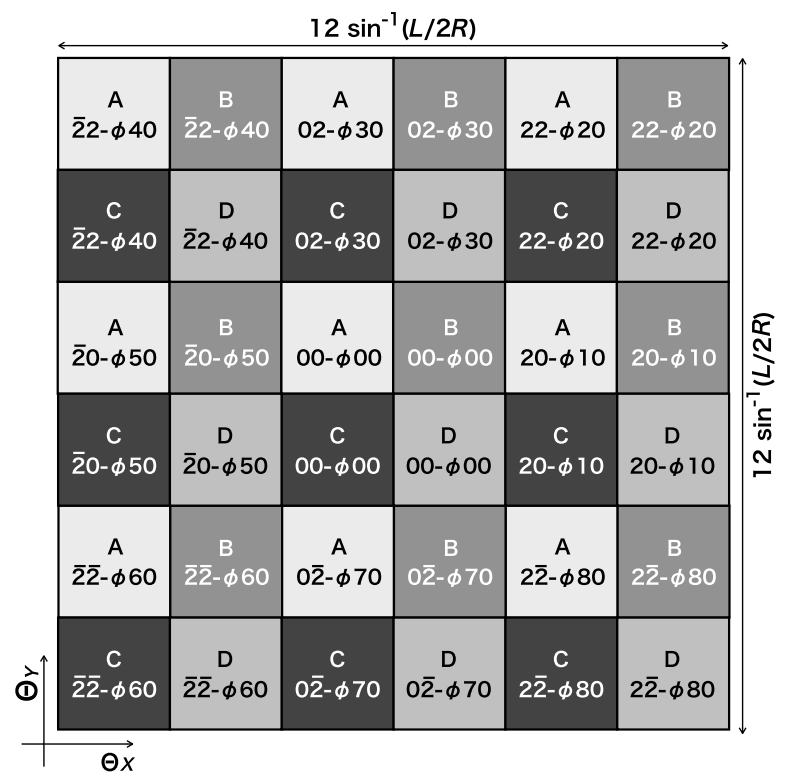

Fig. 4 FoV covered with four units of the nine-segment MuLE optics. A to $D$ denote the unit numbers. For $R=60 \mathrm{~cm}$ and $L=10 \mathrm{~cm}$, the angular span of $57.4 \operatorname{deg} \times 57.4 \mathrm{deg}(\sim 1 \mathrm{sr})$ of the sky is covered with $36 \mathrm{LE}$ segments and four image sensors.

nine-segment MuLE are used, a sky area of $57.4 \mathrm{deg} \times 57.4$ deg can be covered, as shown in Fig. 4. Each unit is named A to D, and an imager is installed directly under ID00 of each unit. The four ID00s of units A to D are installed offset from each other by $2 \theta$ in the $x$ and $y$ directions. Since each segment of the nine-segment MuLE covers the FoV every $4 \theta$ (Fig. 2), it is possible to continuously cover the FoV with four units of nine-segment MuLE. Accordingly, we can achieve an FoV of $\sim 1$ sr with only four imagers (with the total imager area of $10 \times 10 \mathrm{~cm}^{2}$ ).

\section{Ray Tracing Simulation}

To evaluate the performance of the MuLE optics, including the surface roughness of the mirrors, misalignment of the LE mirror cells, and a realistic detector configuration, we performed a ray tracing simulation by modifying a previously built simulator. ${ }^{19}$ The values of the surface roughness and the mirror cell misalignment were taken from our past mirror fabrication. ${ }^{20}$ In this study, we treated only one parameter set because we aimed to evaluate the working principle of the MuLE optics. The optimization of the parameters will be discussed in our next publication. 


\subsection{Simulation Setup}

The simulation was performed with the nine-segment MuLE configuration shown in Fig. 3. One LE segment has a size of $10 \times 10 \mathrm{~cm}^{2}$. Given that the support structures of the LE segments are necessary in a realistic design, a $0.5-\mathrm{cm}$ margin was added around each LE segment. Thus, the geometrical area of each LE segment becomes $9 \times 9 \mathrm{~cm}^{2}$. The nine LE segments are tiled on a spherical surface with a radius $R=60 \mathrm{~cm}$. The azimuthal rotation angle of each LE segment is incremented by $10 \mathrm{deg}$ from $0 \mathrm{deg}$ (central one) to $80 \mathrm{deg}$, as shown in Fig. 3.

Recently, some x-ray mirrors have been produced with a silicon-microelectromechanical systems ( $\mathrm{Si}-\mathrm{MEMS})$ technology ${ }^{20}$ that is a precise and a less expensive technique applicable to the LE optics. We assumed the use of the Si-MEMS technology in the MuLE optics and chose parameters that could be used for manufacturing with current technologies. The thickness of the silicon wafer was $300 \mu \mathrm{m}$, and the pore size was $20 \times 20 \mu \mathrm{m}^{2}$. Since the spacing between adjacent pores was $40 \mu \mathrm{m}$, the open fraction of the aperture was $25 \%$. To keep the structural strength of the Si-MEMS mirror, radial spokes with widths of $300 \mu \mathrm{m}$ were added every $15 \mathrm{deg}$. This reduced the aperture ratio to $21 \%$. Compared with the standard LE mirror made of glass material, the thickness is about one-third, but the other properties such as the point spread function are comparable.

In the simulations, $\mathrm{x}$-rays originating from the nine LE segments were captured by a $4 \mathrm{k} \times$ $4 \mathrm{k}$ pixel imager with a sensitive area of $6.144 \times 6.144 \mathrm{~cm}^{2}$ (i.e., with a $15-\mu \mathrm{m}$ pixel size) centered at the focal point of $f=30 \mathrm{~cm}$. The state-of-art complementary metal-oxide semiconductor (CMOS) technology allows us to use low-noise pixel imagers without cooling. GPixel's CMOS sensors represent these types of devices. ${ }^{21}$ The $15-\mu \mathrm{m}$ pixel size corresponds to the arc length of 10 arcsec in the sky coordinate system. It is small enough compared with the imaging quality of the Si-MEMS LE optics. The detailed values of parameters for the ray tracing simulation are summarized in Table 1.

Table 1 Parameters of the ray tracing simulation.

\begin{tabular}{|c|c|}
\hline Parameter & Value \\
\hline Scan energy $(E)$ & 0.5 to $3.5 \mathrm{keV}$ ( $0.5 \mathrm{keV}$ step) \\
\hline Scan angle of photons $\left(\Theta_{x}, \Theta_{y}\right)^{a}$ & 0 deg to 10 deg (2-deg step) \\
\hline Thickness of LE mirror $(\ell)$ & $300 \mu \mathrm{m}$ \\
\hline Radius of LE sphere $(R)$ & $60 \mathrm{~cm}$ \\
\hline Focal length of LE optics $(f)$ & $30 \mathrm{~cm}$ \\
\hline Open fraction of cells $(\eta)^{\mathrm{b}}$ & 0.21 \\
\hline Cell size $(w \times w)$ & $20 \times 20 \mu \mathrm{m}^{2}$ \\
\hline LE mirror segment size $(L \times L)$ & $10 \times 10 \mathrm{~cm}^{2}$ \\
\hline LE mirror effective area $\left(L_{e} \times L_{e}\right)$ & $9 \times 9 \mathrm{~cm}^{2}$ \\
\hline Mirror coating material & $\mathrm{Pt}$ \\
\hline Mirror surface roughness ${ }^{c}$ & $1 \mathrm{~nm}(\mathrm{rms})$ \\
\hline Mirror point spread function ${ }^{c}$ & $10 \operatorname{arcmin}(\mathrm{FWHM})$ \\
\hline Imager size ${ }^{d}$ & $6.144 \times 6.144 \mathrm{~cm}^{2}$ \\
\hline
\end{tabular}

${ }^{\text {aScan }}$ angles are measured from the center of the FoV of each LE segment.

bShadows induced by the radial spokes are included.

${ }^{\mathrm{c}}$ These values were obtained from our Si-MEMS manufacturing experience.

${ }^{\mathrm{d}} 4 \mathrm{k} \times 4 \mathrm{k}$ square pixels (pixel size $=15 \mu \mathrm{m}$ ). 


\subsection{Image Response of a Point Source}

First, we simulated the image response of a point source focused by the ID00- $\phi 00 \mathrm{LE}$ segment. Figure 5(a) shows the image response of a point source with an incident angle of $\Theta_{x}=\Theta_{y}=$ 0 deg with respect to the central optical axis of the ID00- $\phi 00$ segment. Approximately $15 \%$ of the detected photons at $0.6 \mathrm{keV}$ were scattered twice on the adjacent walls of the LE cells and focused at the center of the imager (marked as "Focus" in the figure). Approximately $48 \%$ of the photons were scattered once on the cells and concentrated in the cross-like arm foci (these are marked as "ArmX" and "ArmY"). The remaining 37\% of the photons were dropped through the cells directly to the imager (these are marked as "NoRef"). The boundary limit angle beyond which NoRef photons do not exist is defined by $\theta_{\lim }=\tan ^{-1}(w / \ell)=3.81 \mathrm{deg}$ and corresponds to $4 \mathrm{~cm}\left(=R \sin \theta_{\text {lim }}\right)$ on the imager.

Second, we considered the point source with an incident angle of $\Theta_{x}=4 \mathrm{deg}$ and $\Theta_{y}=0$ deg with respect to the central optical axis of ID00. Figure 5(b) shows that the image of the source clearly shifted to the right compared with Fig. 5(a). Only half of the image was detected in the x-axis direction, but it was sufficiently detected even at the edge of the FoV. In realistic configurations used in x-ray astronomy, the missing half of the x-ray images could be detected by another MuLE unit given that the FoVs are tiled without gaps, as shown in Fig. 4, i.e., the reduction of the effective area can be almost mitigated. The boundary created by the edge of the LE segment is clearly seen in Fig. 5 (b) at $X_{\operatorname{det}}=2.4 \mathrm{~cm}$ for $\Theta_{x}=4 \mathrm{deg}$. See Appendix A for a detailed description of the edge of the LE segment.

Finally, we simulated the point source images focused by the ID20 and ID22 segments. Figure 5(c) shows the image focused by the ID20- $\phi 10$ segment. The image response was similar to that of ID00 but was rotated $10 \mathrm{deg}$ as the LE segment rotated. Figure 5(d) shows the image
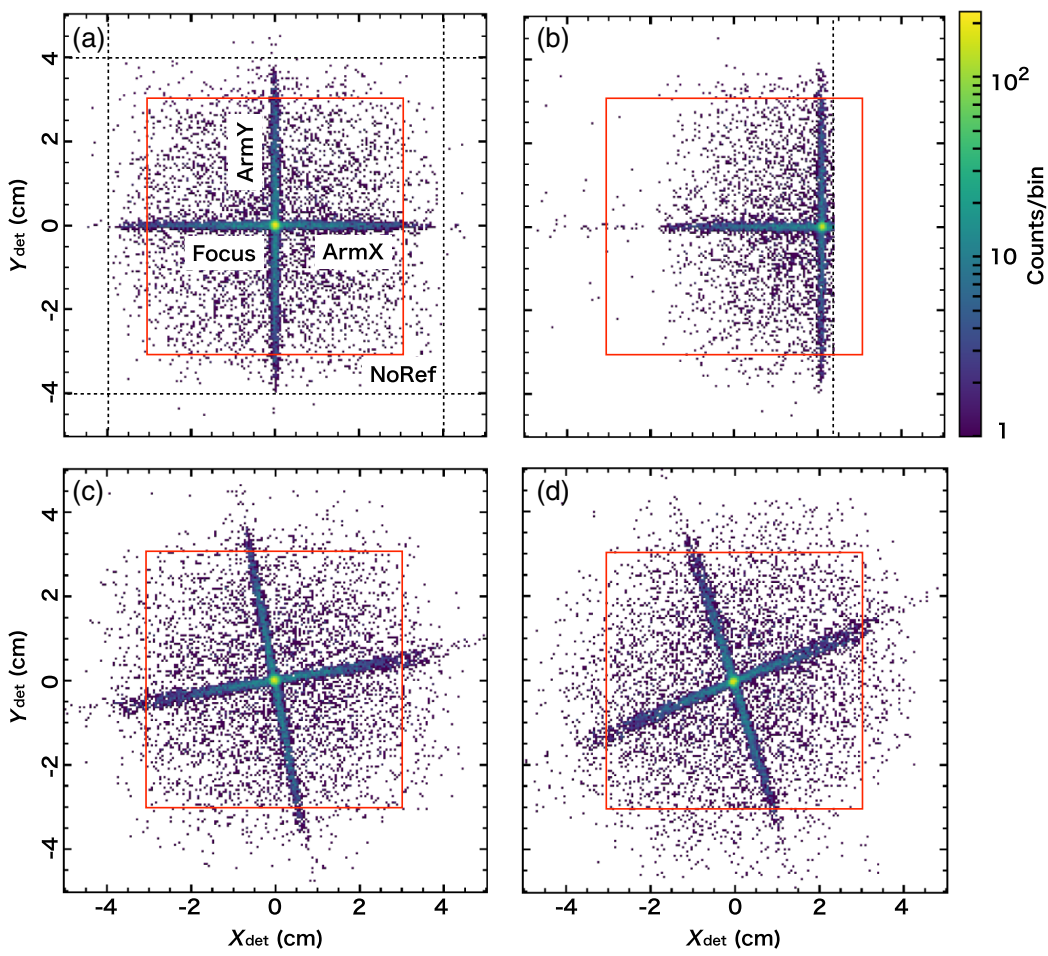

Fig. 5 (a) Simulated image of a point source focused by ID00- $\phi 00$ with an incident photon angle of $\Theta_{x}=\Theta_{y}=0$ deg. See text for detail on "Focus" and "ArmX/Y." The dashed lines show the boundary beyond which there are not non-reflected (NoRef) photons. The red rectangle shows the size of the imager. (b) ID00- $\phi 00$ with $\Theta_{x}=4 \mathrm{deg}$ and $\Theta_{y}=0 \mathrm{deg}$. The dashed line shows the image boundary produced by the edge of the LE segment. (c) ID20- $\phi 10$ with $\Theta_{x}=\Theta_{y}=0$ deg. (d) ID22- $\phi 20$ with $\Theta_{x}=\Theta_{y}=0$ deg. The color bar shows the counts-per-bin on a logarithmic scale. All of the images were reconstructed with $\sim 12,000$ photons at $0.6 \mathrm{keV}$. 
focused by the ID22- $\phi 20$ segment. The cross-like images in both the ID20 and ID22 segments were clearly seen. This implied that the images from any LE segment could be detected.

As expected, defocus aberration was observed at the edge of the CMOS image sensor for the ID20 and ID22 segments, given that the focal plane was tilted in these segments. The worst case of the defocus aberration appeared at the diagonal edge of the CMOS imager for the ID22 segment. At that point, the focal length was $\sim 1.1 \mathrm{~cm}$ shorter than that for the true focal length $f=30 \mathrm{~cm}$. The defocus corresponds to a \pm 8.0 arcmin aberration in the sky coordinate system. Since this is almost comparable to the FWHM size $(10 \mathrm{arcmin})$ of a point source focused by the $\mathrm{Si}-\mathrm{MEMS}$ mirrors, the defocus was not a problem in our configuration.

\subsection{Effective Area}

The mirror effective areas were also derived from the ray tracing simulation. Figures 6(a)-6(d) show the effective areas of ID00 as a function of the incident photon angle measured from the optical axis of the LE segment for $0.5,1.0,2.0$, and $3.5 \mathrm{keV}$, respectively. In this calculation, the size of the CMOS sensitive area was taken into account, but the quantum efficiency of the imager was not since the efficiency is almost $100 \%$ in this energy band. The simulations were conducted based on discrete calculations within the angle range of $\Theta_{x}$ at 2 deg steps. The reason for including NoRef in the figures of the effective areas was that the LE optics had two functions: a focusing mirror (ArmX/Y and Focus) and a collimator (NoRef). Since the density of x-ray objects in the sky is sparse, if no other object is in the FoV, NoRef is identified as x-rays from the target object.

The curved lines shown in Fig. 6 are the analytically calculated effective area in combination with the mirror reflectivity. ${ }^{22}$ The detailed procedure of the analytic calculation is summarized in Appendix B. The discontinuity marked (i) in Fig. 6(a) shows the angle where the Focus is shifted off the edge of the CMOS. The effective area for ID20 and ID22 at $0.5 \mathrm{keV}$ is shown in Figs. 7(a) and 7(b) respectively. Given that the difference between ID20/22 and ID00 is originated only in

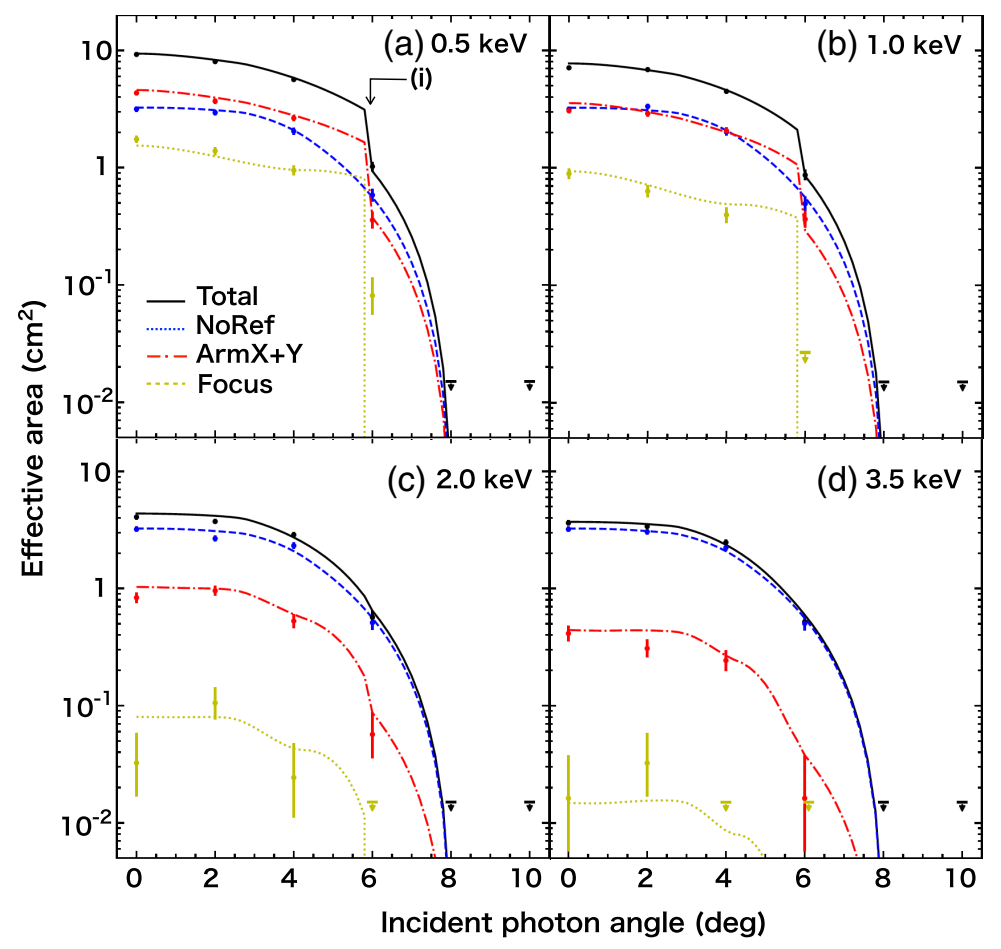

Fig. 6 Effective area as a function of the incident photon angle $\Theta_{x}$ with $\Theta_{y}=0$ deg for ID00 at (a) 0.5 , (b) 1.0 , (c) 2.0, and (d) $3.5 \mathrm{keV}$. Only the photons collected by the CMOS imager are taken into account. The data points show the results of the ray tracing simulation, and the curves show the analytic calculation. Point (i) indicates the edge angle where the Focus is shifted off the edge of the image sensor. 


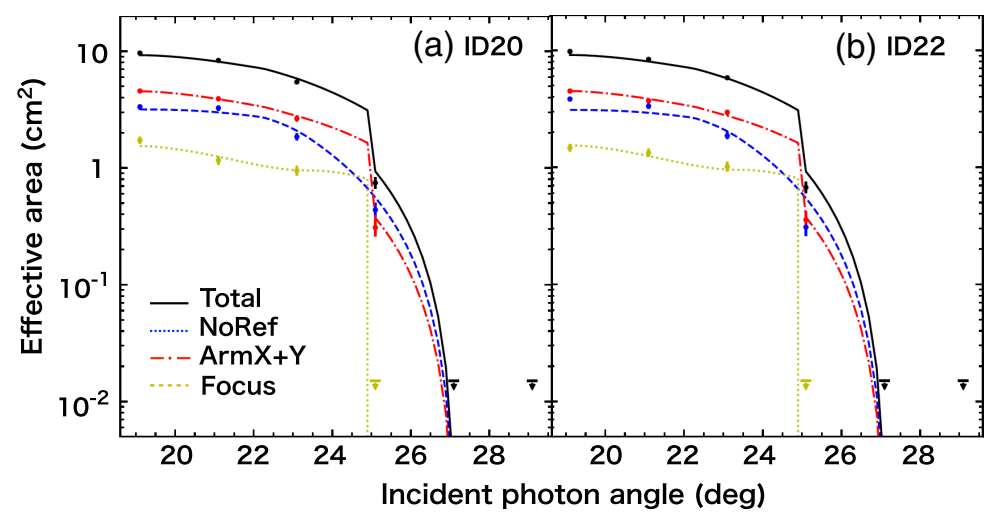

Fig. 7 Effective area as a function of the incident photon angle $\Theta_{x}$ for (a) ID20 with $\Theta_{y}=0$ deg and (b) ID22 with $\Theta_{y}=19.1 \mathrm{deg}$ at $0.5 \mathrm{keV}$. The angles are measured from the optical axis of ID00. The curves of the effective areas are symmetrical about 19.1 deg.

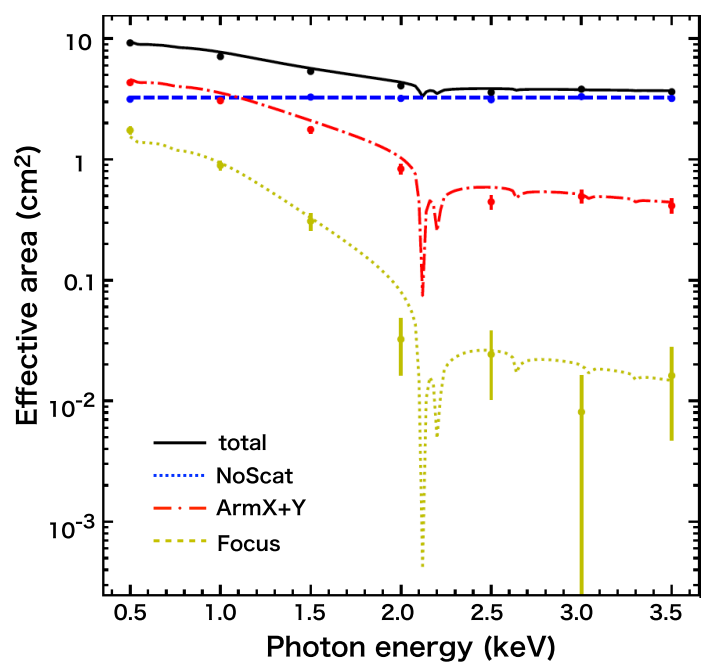

Fig. 8 Effective area as a function of photon energy for ID00 with an incident angle of $\Theta_{x}=\Theta_{y}=0$ deg.

the tilt angle of the x-ray images, the curves of the effective area look very similar to each other. To clarify the characteristics of the nine-segment MuLE optics only, the vignetting is shown in Appendix C.

The effective areas of the mirror as a function of the incident photon energy for the ID00 segment with an incident angle of $\Theta_{x}=\Theta_{y}=0$ deg are shown in Fig. 8. The ray tracing simulation was performed for different energies at every $0.5 \mathrm{keV}$ from 0.5 to $3.5 \mathrm{keV}$. The curves of the effective areas derived from the analytic calculation are also shown in the figure. While the effective area of NoRef was flat, the effective areas of ArmX/Y and Focus dropped rapidly as the energy increased. The effective area of ArmX+Y was somewhat larger than that of NoRef below $1 \mathrm{keV}$.

\subsection{Source Detection Limit}

\subsubsection{Photon and background limit cases}

The source detection limit was determined by the signal-to-noise ratio of the x-ray photons on the imager. In the MuLE optics, the most dominant noise is the diffuse CXB. Figure 9 shows the $5 \sigma$ detection limits for Focus, Focus + ArmX/Y, and total (Focus + ArmX/Y + NoRef) when a point source was located at the center of the ID00 FoV. To extract foreground and background 


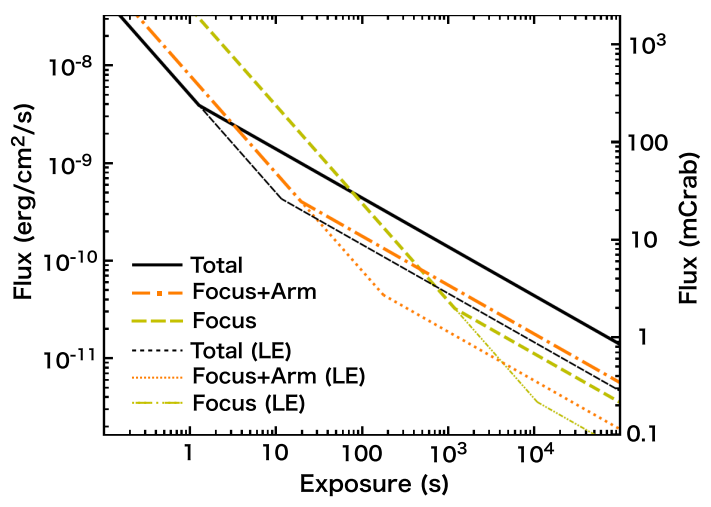

Fig. 9 The $5 \sigma$ detection limit of a point source at the center of the ID00 FoV in the 0.5 to $2.0 \mathrm{keV}$ bandpass for the nine-segment MuLE optics. The $5 \sigma$ detection limit for the standard LE configuration is overlaid.

photons in the region of ArmX $+\mathrm{Y}$ and Focus, we selected the photons in the strip regions along the arm foci (widths of $0.2 \mathrm{~cm}$ ). The strip width was not optimized but was adequately large enough to collect the photons focused by the LE segments even in the cases in which the image suffered defocus. Throughout this study, we assumed a Crab-like spectrum ${ }^{23}$ for a point source characterized by a power-law photon index of 2.07 , normalization of 8.26 photons $\mathrm{keV}^{-1} \mathrm{~cm}^{-2} \mathrm{~s}^{-1}$ at $1 \mathrm{keV}$, and an absorption of $N_{H}=4.5 \times 10^{21} \mathrm{~cm}^{-2}$.

The flux limit was governed by the number of photons for shorter exposures (photon limit) and was proportional to $t^{-1}$, where $t$ is the exposure time. Conversely, the flux limit was governed by the CXB photons in the cases of longer exposures (background limit) and was proportional to $t^{-0.5}$ because the number of background photons obeyed Poisson's law.

Figure 9 also shows the $5 \sigma$ detection limits for the standard LE configuration in which the size and properties of the LE segments were exactly the same but the images were not multiplexed. Mathematically, the amount of background was reduced to one-ninth from that of the MuLE configuration. The difference between the two configurations only appears in the background limit case as shown in Fig. 9.

\subsubsection{Confusion case of background point source}

Another possible weak point relevant to the nine-segment MuLE configuration is its large FoV, which causes contamination of bright background sources in the imager. The detection ability of a faint source is easily affected by a bright background source located in any of the nine FoVs. We considered a background point source that was 0.5 deg away from the object, which we

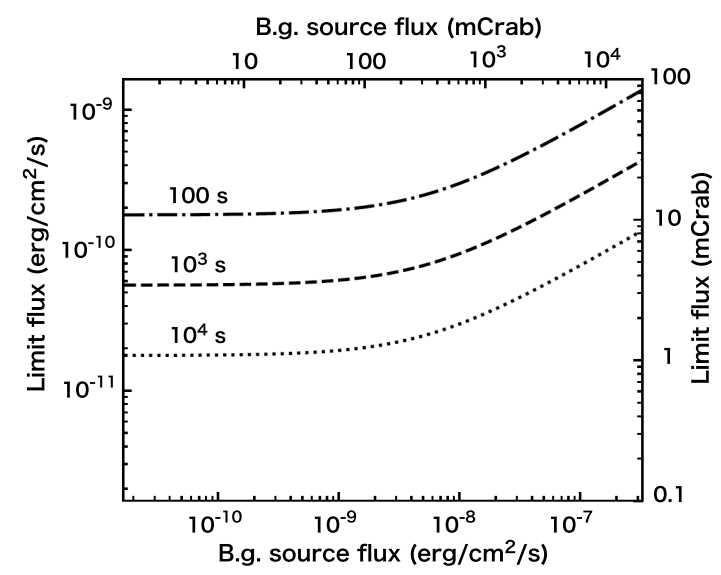

Fig. 10 Degradation of the $5 \sigma$ detection limit in the 0.5 to $2 \mathrm{keV}$ band owing to the background (b.g.) source of the $0.5-\mathrm{deg}$ separation distance in the case of the nine-segment MuLE optics. 
observed at the center of the FoV to evaluate its effects. We calculated the detection limit change owing to the bright object for the case of Focus + ArmX/Y described in Fig. 9.

Figure 10 shows the detection limits for the observation times of $100,10^{3}$, and $10^{4} \mathrm{~s}$. When the brightness of the background point source was brighter than $100 \mathrm{mCrab}$, the detection limit was degraded. This is because the flux limit was governed by the CXB, which is almost equivalent to a $100-\mathrm{mCrab}$ source.

There are $~ 30$ objects in the entire sky that are brighter than $100 \mathrm{mCrab}$ in the $\mathrm{x}$-ray band. For the nine-segment MuLE configuration that we considered, the FoV was about $666 \mathrm{deg}^{2}$ (nine $8.6 \mathrm{deg} \times 8.6 \mathrm{deg}$ FoVs), which corresponds to $1.6 \%$ of the entire sky and contains $\sim 0.5$ bright objects on average. Since many bright $\mathrm{x}$-ray objects are distributed along the galactic plane, they are not a fatal background when we observe the region of high galactic latitudes.

\subsection{FoV Discrimination Based on Image Response}

To evaluate the power of the FoV discrimination by the cross-like image response, we also employed the ray tracing simulation. This problem is converged to a problem that pertained to the determination of the azimuthal rotation angle of the cross-like image.

\subsubsection{Method used to determine the azimuthal rotation angle of cross-like image}

We considered the nine-segment MuLE configuration shown in Fig. 3. When the flux from a transient object exceeds the detection limit, at least one image is captured. At this moment, it is unclear which LE segment (ID00- $\phi 00$ to ID2 $\overline{2}-\phi 80$ ) focused the image. In consideration of all possibilities, the image is subjected to nine different operations to identify the LE segment that was involved. The procedure that we employed is as follows.

(1) For LE segments other than ID00, image distortion should be corrected first given that the imager was tilted with respect to the tangential plane at the center of the LE segment. The distortion correction produced eight different images. Details of the correction are described in Appendix D. Currently, there are a total of nine images.

(2) By identifying the center of gravity of the entire photons, the position of the transient source on the imager $\mathrm{O}_{i}(i=1, \ldots, 9)$ is determined in all nine images.

(3) The position of each photon $\mathrm{P}_{i, m}(m=1, \ldots, N)$ is recorded, where $N$ is the number of total photons. Then, the azimuthal rotation angles $\phi_{i, m}$ of the vector from $\mathrm{O}_{i}$ to $\mathrm{P}_{i, m}$ are calculated. The azimuthal rotation angles $\phi_{i, m}$ are measured from the azimuthal rotation angle $\phi_{i}$ of the square cells of the LE segment.

(4) The azimuthal rotation angles $\phi_{i, m}$ are filled in a histogram between $-45 \mathrm{deg}$ and +45 deg given that the cross-like point source image has four-fold rotational symmetry. Only the photons in a ring region of the radius between 0.15 and $3 \mathrm{~cm}$ are sampled concentrically around $\mathrm{O}_{i}$. Figure 11 shows an example of the histogram for the case of ID00- $\phi 00$. Herein, there are a total of nine histograms.

(5) The point source responses prepared in advance for all nine LE segments are fitted to a histogram, and the goodness-of-fit was found based on the maximum likelihood estimation. The point source response is generated by the ray tracing simulation with sufficient statistics for more than 100,000 photons: CXB photons are not included. The response was modeled with a Lorentzian function and a constant according to

$$
f(x)=S \frac{\Gamma / 2}{\left(\phi-\phi_{i}\right)^{2}+(\Gamma / 2)^{2}}+N .
$$

The parameter $\phi_{i}$ was fixed to the azimuthal rotation angle of the LE segment cells, and the half-width was fixed to the value $\Gamma / 2=1.975$ deg derived from the simulation. The other two parameters, Lorentzian normalization $S$ and the constant value $N$, were free in the fit. An example of the fit is shown in Fig. 11. 


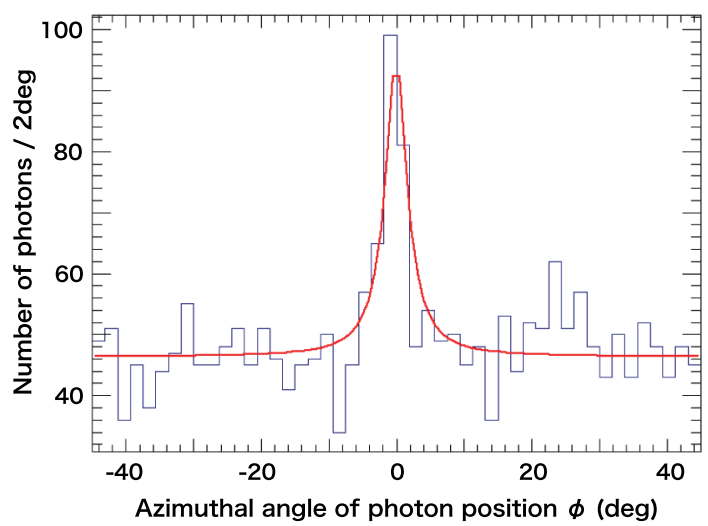

Fig. 11 (Histogram) Typical distribution of the azimuthal angles of photon positions for the 250 source and $100 \mathrm{~s}$ CXB photons for the nine-segment MuLE optics. (Curve) The best fit result of the plotted distribution with the template response.

(6) The operations are performed for all nine images, and the one with the highest $S / N$ is selected as the LE segment from which the point source originated.

\subsubsection{FoV determination for IDOO and ID22}

We performed ray tracing simulations for a transient with a duration of $100 \mathrm{~s}$ to evaluate if we could localize its position as a function of the source flux. In this study, the number of CXB photons was fixed for $100 \mathrm{~s}$ observation, but the number of $\mathrm{x}$-ray photons from the transient source was varied. Using the method described in Sec. 3.5.1, the segment that focused the photons on to the imager was determined from the simulated data. Figure 12 shows the fraction associated with the selection of the correct LE segment as a function of the number of source photons. Each data point was the average of 350 to 850 trials. Corresponding error bars are also plotted. For simplicity, this study was conducted with $0.6 \mathrm{keV}$ photons.

The number of photons required to achieve $95 \%, 99 \%$, and $99.7 \%$ correct outcome rates for ID00 were 142, 182, and 212, respectively. In combination with Fig. 9, the position of a point source was correctly determined in $97 \%$ of the events at the $5 \sigma$ detection threshold for $100 \mathrm{~s}$ observations. Even if the correct LE segment could not be determined from the data, the source

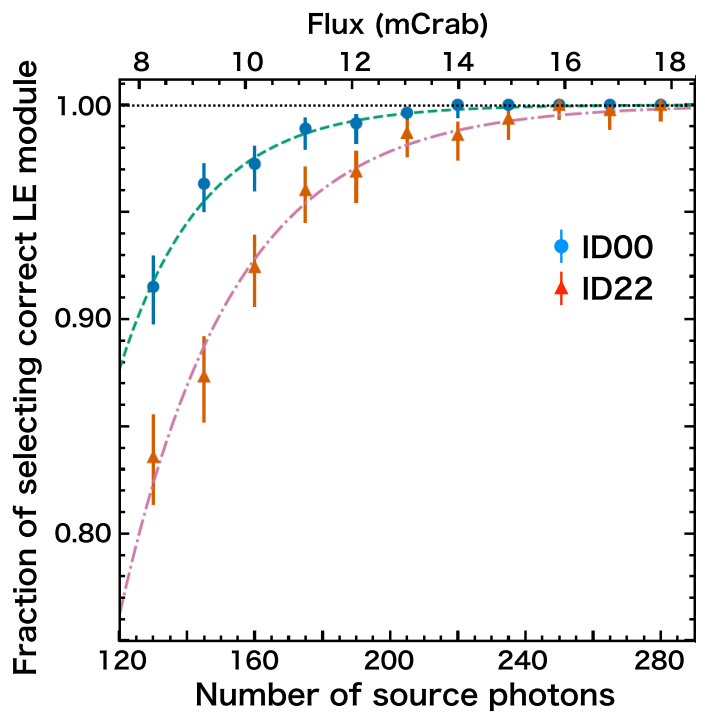

Fig. 12 Fraction associated with the selection of the correct LE segment for 0.6-keV x-rays detected with the nine-segment MuLE optics. 
position on the imager was determined with an FWHM accuracy of $\sim 10$ arcmin and can be narrowed down to nine points in the sky coordinate system. Furthermore, if the correct LE segment can be identified with a deep learning approach, the determination accuracy may be improved.

Similarly, the number of photons required to achieve $95 \%, 99 \%$, and $99.7 \%$ of correct outcome rates for ID22 were 172, 226, and 266, respectively. The reason for the correct outcome rate being lower than that of ID00 at the same photon numbers is that the image sensor of ID22 was tilted with respect to the tangential surface of the LE segment and the arm of the cross-like response was blurred owing to the defocus effect. It would be useful to perform a more detailed survey to assess the performance of the MuLE optics. However, this is beyond the scope of this study and will be described in our next publication.

\section{Discussions and Outlook}

This study described the working principle of the MuLE optics in which multiple LE segments focused x-rays onto a single imager. This configuration reduced the number of image sensors considerably and thus overcame a disadvantage of the LE optics. A ray tracing simulation was performed to evaluate the properties of the MuLE optics based on the assumption of a ninesegment configuration. In the simulation, only existing technologies ( $\mathrm{Si}-\mathrm{MEMS}$ mirrors and a CMOS image sensor) that will help with the construction of an inexpensive and accurate enough wide-field $x$-ray monitor in the near future were assumed.

When the focal length of $30 \mathrm{~cm}$ and an area spanning $9 \times 9 \mathrm{~cm}^{2}$ of an LE segment were used, the total effective area at $1 \mathrm{keV}$ was calculated to be $8 \mathrm{~cm}^{2}$ at the center of the FoV, and about $4 \mathrm{~cm}^{2}$ at the edge of the FoV $(\Theta= \pm 4.3 \mathrm{deg})$. The $5 \sigma$ detection limit in the 0.5 - to $2-\mathrm{keV}$ band for a transient with a duration of $100 \mathrm{~s}$ at the center of the FoV was $\sim 2 \times 10^{-10} \mathrm{erg} \mathrm{cm}^{-2} \mathrm{~s}^{-1}$ (10 mCrab). The ability to determine the correct position achieved a $99.7 \%$ level for a 14 to $17 \mathrm{mCrab}$ point source with a duration of $100 \mathrm{~s}$. Thus, we finally conclude that the MuLE optics can be used to implement a wide FoV transient monitor with sufficient sensitivity.

Given that the MuLE configuration is the easiest way to reduce considerably the number of image sensors, it is considered to be effective for a small satellite with limited resources or a small observatory on-board the International Space Station. With the use of the three units of the nine-segment MuLE with $f=30 \mathrm{~cm}$, as presented in this study, it is possible to cover a $0.75 \mathrm{sr}$ of an FoV with a microsatellite with a volume of $50 \times 50 \times 50 \mathrm{~cm}^{3}$. With 16 satellite sets, the entire sky can be covered. Using lightweight and inexpensive Si-MEMS technology and by reducing the number of imaging devices with MuLE, the price per MuLE unit can be reduced considerably. Accordingly, the establishment of a constellation of these types of microsatellites is possible.

The ability to cover the entire sky at all times with the satellite constellation will have a major impact in the multimessenger and time-domain astronomy. If the focal length is reduced by half to $15 \mathrm{~cm}$, the number of satellites in the constellation can be reduced to four, though the sensitivity will drop. In addition, given that the MuLE configuration that we described in this study can achieve about $1 \mathrm{mCrab}$ at $10^{4} \mathrm{~s}$, it can be used as an all-sky monitor, such as MAXI or RXTE/ASM. Since the position is known in advance, for a known source, it is not necessary to identify the azimuthal rotation angle of the cross-like image, and the point source can be determined using only the location on the image sensor. By optimizing the parameters, such as an increase in the thickness of the Si-MEMS mirror, we can fabricate more sensitive all-sky monitors. In a future publication, we will discuss parameter optimization and examine the detailed performance of those configurations.

\section{Appendix A: Boundaries in the Lobster-Eye Optics}

Since the LE segments and LE hollow cells have a finite size, various boundaries appear in the LE optics. Here, we explain the origins of some important boundaries. For the specific numerical values shown in this section, the same parameters used in the simulation were used. Figure 13(a) shows the definition of the LE FoV. It is defined that the center of the cross-like image is exactly 
(a)
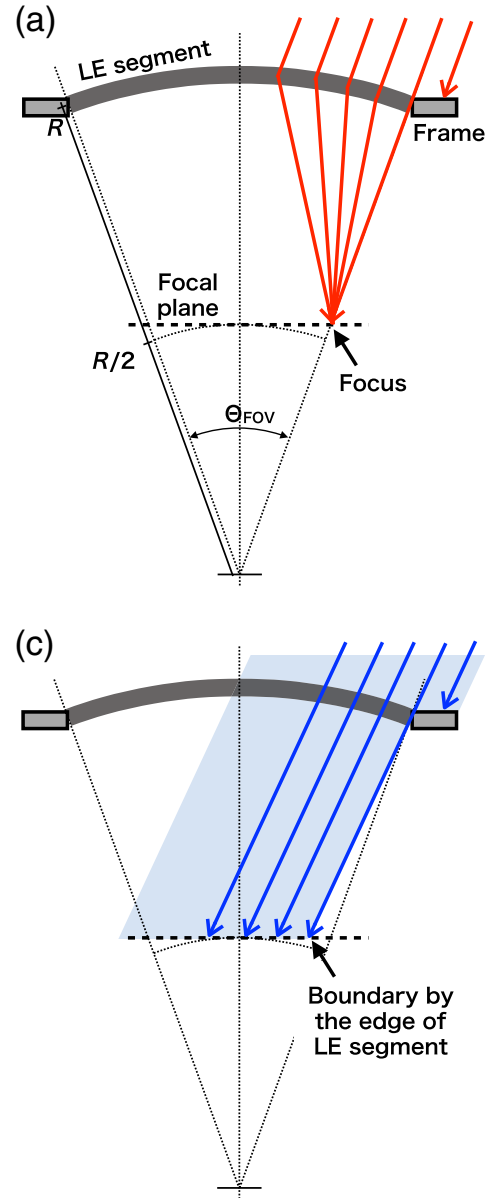

(b)

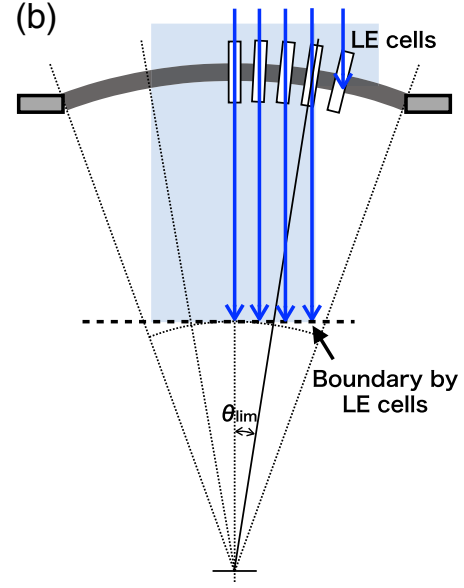

Fig. 13 (a) The definition of the FoV of the LE optics. (b) The boundary produced by the LE cells and the limit angle $\theta_{\mathrm{lim}}$. (c) The boundary produced by the edge of the LE segment. These figures are exaggerated for readability. The boundaries (b) and (c) are common for the NoRef photons and the unfocused direction of the Arm foci.

on the line connecting the edge of the LE segment and the center of curvature. With the parameters used in our simulation, the FoV becomes $\Theta_{\mathrm{FOV}} \sim L_{e} / R \mathrm{rad}=8.6 \mathrm{deg}$. Figure 13 (b) shows the boundary limited by the LE hollow cells for the photons that pass through without reflection. This is the boundary visible in Fig. 5(a). With our LE parameter, the limit angle becomes $\theta_{\lim }=\tan ^{-1}(w / \ell)=3.81 \mathrm{deg}$. Figure 13 (c) shows the boundary limited by the support structure (frame) of the LE segment for the photons that pass through without reflection. There are no nonreflected photons outside the boundary as seen in Fig. 5(b). As observed from Fig. 13(c), the location of this boundary is a function of the incident photon angle.

\section{Appendix B: Analytic Estimation of the Effective Area}

We summarize herein the methodology to calculate the effective areas. When $\mathrm{x}$-ray photons enter a cell of an LE segment, some of them go through the cell without reflection; the others are reflected by the wall of the cell once, twice, or more times. These photons can be categorized by the number of reflections. ${ }^{13}$ The fraction of each category is a function of the tilt angle of a cell $\theta_{j}$, where $j$ denotes $x$ or $y$. In the case for which there are no reflections, the fraction is

$$
f_{0}^{i}\left(\theta_{j}\right)=\left\{\begin{array}{ll}
1-\frac{\ell}{w} \tan \left(\theta_{j}\right) & \theta_{j} \leq \tan ^{-1}\left(\frac{w}{\ell}\right) \\
0 & \theta_{j}>\tan ^{-1}\left(\frac{w}{\ell}\right)
\end{array} .\right.
$$


In the case for which there is a single reflection

$$
f_{1}^{j}\left(\theta_{j}\right)= \begin{cases}\frac{\ell}{w} \tan \left(\theta_{j}\right) & \theta_{j} \leq \tan ^{-1}\left(\frac{w}{\ell}\right) \\ 2-\frac{\ell}{w} \tan \left(\theta_{j}\right) & \tan ^{-1}\left(\frac{w}{\ell}\right)<\theta_{j} \leq \tan ^{-1}\left(\frac{2 w}{\ell}\right) . \\ 0 & \theta_{j}>\tan ^{-1}\left(\frac{2 w}{\ell}\right)\end{cases}
$$

In our setup shown in Table 1 , the boundary angles are $\tan ^{-1}(w / \ell)=3.81 \mathrm{deg}$ and $\tan ^{-1}(2 w / \ell)=7.59 \mathrm{deg}$.

Using the photon fraction sorted by the number of reflections, the effective areas are derived as follows:

$$
\begin{gathered}
A_{\text {NoRef }}\left(\Theta_{x}, \Theta_{y}\right)=\frac{A \eta}{N^{x} N^{y}} \int_{\theta_{x}^{\min }}^{\theta_{x}^{\max }} f_{0}^{x} \mathrm{~d} \theta_{x} \int_{\theta_{y}^{\min }}^{\theta_{y}^{\max }} f_{0}^{y} \mathrm{~d} \theta_{y}, \\
A_{\text {ArmX }}\left(E, \Theta_{x}, \Theta_{y}\right)=\frac{A \eta}{N^{x} N^{y}} \int_{\theta_{x}^{\max }}^{\theta_{x}^{\min }} f_{0}^{x} \mathrm{~d} \theta_{x} \int_{\theta_{y}^{\max }}^{\theta_{y}^{\min }} \xi\left(E, \theta_{y}\right) f_{1}^{y} \mathrm{~d} \theta_{y}, \\
A_{\text {ArmY }}\left(E, \Theta_{x}, \Theta_{y}\right)=\frac{A \eta}{N^{x} N^{y}} \int_{\theta_{x}^{\min }}^{\theta_{x}^{\max }} \xi\left(E, \theta_{x}\right) f_{1}^{x} \mathrm{~d} \theta_{x} \int_{\theta_{y}^{\max }}^{\theta_{y}^{\min }} f_{0}^{y} \mathrm{~d} \theta_{y}, \\
A_{\text {Focus }}\left(E, \Theta_{x}, \Theta_{y}\right)=\frac{A \eta}{N^{x} N^{y}} \int_{\theta_{x}^{\max }}^{\theta_{x}^{\min }} \xi\left(E, \theta_{x}\right) f_{1}^{x} \mathrm{~d} \theta_{x} \int_{\theta_{y}^{\max }}^{\theta_{y}^{\min }} \xi\left(E, \theta_{y}\right) f_{1}^{y} \mathrm{~d} \theta_{y},
\end{gathered}
$$

where $A$ is the geometrical area $L_{\mathrm{e}} \times L_{\mathrm{e}}, \eta$ is the open fraction of the pore, $N^{j}$ is the normalization factor $\int \mathrm{d} \theta_{j}$, and $\xi\left(E, \theta_{j}\right)$ is the reflectivity of the platinum-coated LE mirror with a surface roughness of $1 \mathrm{~nm}$ that refers to the x-ray database of the Lawrence Berkeley National Laboratory. ${ }^{22}$ The limit angles $\theta_{j}^{\max }$ and $\theta_{j}^{\min }$ are restricted by the edge of the LE segment, including the radial spokes and the CMOS sensor. The limit angles vary as the incident photon angles $\Theta_{x}$ and $\Theta_{y}$ vary because the viewing angle of the edge changes.

\section{Appendix C: Vignetting of the MuLE Optics}

We showed the effective areas of the MuLE configuration in Figs. 6 and 7, but they contain the effect by the finite size of the imaging detector. It is worthwhile to show here the vignetting of the MuLE optics. Figure 14 shows the vignetting curve along $\Theta_{x}$ with $\Theta_{y}=0$ deg for $0.5 \mathrm{keV}$ photons. The second peak centered at $19.1 \mathrm{deg}$ was due to the ID20 segment.

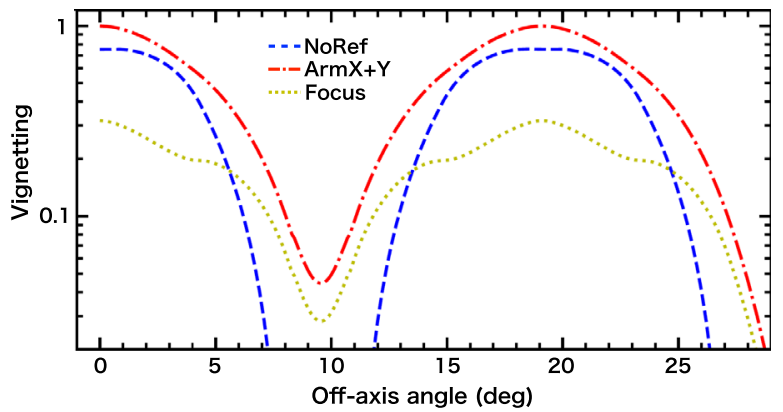

Fig. 14 Vignetting curve of the nine-segment MuLE optics as a function of off-axis angle along $\Theta_{x}$ with $\Theta_{y}=0$ for $0.5 \mathrm{keV}$ photons. The vignetting is normalized by the value of $\operatorname{Arm} X+Y$ at 0 deg. 


\section{Appendix D: Correction Method of Elongated Images Detected with a Tilted Imager}

The images focused by any LE segment-except the ID00-are elongated because the focal plane imager is tilted with respect to the true focal plane of each LE segment. To correct the elongated images, the following operation should be applied:

$$
\left(\begin{array}{l}
x^{\prime} \\
y^{\prime}
\end{array}\right)=A^{-1}\left(\begin{array}{l}
x \\
y
\end{array}\right)
$$

where $(x, y)$ is the original position of a photon on an imager and $\left(x^{\prime}, y^{\prime}\right)$ is the corrected position of the photon if the imager is located at the proper focal plane of the LE segment without the tilt angle. The matrices $A$ are defined as

$$
A=\left(\begin{array}{ll}
k & 0 \\
0 & 1
\end{array}\right), \quad\left(\begin{array}{ll}
1 & 0 \\
0 & k
\end{array}\right), \quad\left(\begin{array}{cc}
\frac{k^{\prime}+1}{2} & \frac{k^{\prime}-1}{2} \\
\frac{k^{\prime}-1}{2} & \frac{k^{\prime}+1}{2}
\end{array}\right), \quad \text { and } \quad\left(\begin{array}{cc}
\frac{k^{\prime}+1}{2} & \frac{-k^{\prime}+1}{2} \\
\frac{-k^{\prime}+1}{2} & \frac{k^{\prime}+1}{2}
\end{array}\right)
$$

for ID20- $\phi 10 / \phi 50$, ID02- $\phi 30 / \phi 70$, ID22- $\phi 20 / \phi 60$, and ID22- $\phi 40 / \phi 80$, respectively, where $k=1 / \cos \left(\theta_{t}\right)$ and $k^{\prime}=1 / \cos \left(\theta_{t}^{\prime}\right)$. The tilted angles $\theta_{t}$ and $\theta_{t}^{\prime}$ are defined as $4 \tan ^{-1}(L / 2 R)$ and $4 \tan ^{-1}(\sqrt{2} L / 2 R)$, respectively.

\section{Acknowledgments}

This work was partially supported by the JSPS KAKENHI (Grant No. JP18K18775), Toray Science Foundation, and the budget for basic R\&D onboard equipment for future space science missions by the Advisory Committee for Space Science Japan.

\section{References}

1. R. Vanderspek et al., "HETE observations of the gamma-ray burst GRB 030329: evidence for an underlying soft x-ray component," Astrophys. J. 617, 1251-1257 (2004).

2. B. P. Abbott et al., "GW170817: observation of gravitational waves from a binary neutron star inspiral," Phys. Rev. Lett. 119, 161101 (2017).

3. B. P. Abbott et al., "Multi-messenger observations of a binary neutron star merger," Astrophys. J. Lett. 848, L12 (2017).

4. The IceCube Collaboration, "Multimessenger observations of a flaring blazar coincident with high-energy neutrino IceCube-170922A," Science 361(6398), eaat1378 (2018).

5. Ž. Ivezić et al., "LSST: from science drivers to reference design and anticipated data products," Astrophys. J. Lett. 873(2), 111 (2019).

6. S. T. Ridgway et al., "The variable sky of deep synoptic surveys," Astrophys. J. Lett. 796(1), 53 (2014).

7. C. Winkler et al., "The integral mission," Astron. Astrophys. 411, L1-L6 (2003).

8. H. A. Krimm et al., "The swift/bat hard x-ray transient monitor," Astrophys. J. Suppl. Ser. 209(1), 14 (2013).

9. Y. Shirasaki et al., "Design and performance of the wide-field x-ray monitor on board the high-energy transient explorer 2," Publ. Astron. Soc. Jpn. 55, 1033-1049 (2003).

10. R. Jager et al., "The wide field cameras onboard the BeppoSAX x-ray astronomy satellite," Astron. Astrophys. Suppl. Ser. 125, 557-572 (1997).

11. A. M. Levine et al., "First results from the all-sky monitor on the Rossi x-ray timing explorer," Astrophys. J. Lett. 469, L33-L36 (1996).

12. M. Matsuoka et al., "The MAXI mission on the ISS: science and instruments for monitoring all-sky x-ray images," Publ. Astron. Soc. Jpn. 61, 999-1010 (2009).

13. J. R. P. Angel, “Lobster eyes as X-ray telescopes," Astrophys. J. Part 1 233, 364-373 (1979).

14. W. Yuan et al., "Einstein probe: a lobster-eye telescope for monitoring the X-ray sky," Proc. SPIE 10699, 1069925 (2018). 
15. J. Camp et al., "Using ISS telescopes for electromagnetic follow-up of gravitational wave detections of NS-NS and NS-BH mergers," Exp. Astron. 36, 505-522 (2013).

16. D. Yonetoku et al., "High-z gamma-ray bursts for unraveling the dark ages mission HiZGUNDAM," Proc. SPIE 9144, 91442S (2014).

17. W. C. Priedhorsky, A. G. Peele, and K. A. Nugent, "An x-ray all-sky monitor with extraordinary sensitivity," Mon. Not. R. Astron. Soc. 279, 733-750 (1996).

18. J. E. Trümper, G. Hasinger, and R. Staubert, "ABRIXAS-a broad-band imaging X-ray allsky survey," Astron. Nachr. 319(1-2), 113-116 (1998).

19. I. Mitsuishi et al., "Ray-tracing simulations for the ultra-lightweight x-ray optics toward a future Jupiter exploration mission," Adv. Space Res. 57(1), 320-328 (2016).

20. Y. Ezoe et al., "Ultralightweight X-ray telescope missions: ORBIS and GEO-X," J. Astron. Telesc. Instrum. Syst. 4, 046001 (2018).

21. W. Wang et al., "Developments of scientific CMOS as focal plane detector for Einstein probe mission," Proc. SPIE 10699, 1069950 (2018).

22. B. L. Henke, E. M. Gullikson, and J. C. Davis, "X-ray interactions: photoabsorption, scattering, transmission, and reflection at $\mathrm{E}=50-30000 \mathrm{eV}, \mathrm{Z}=1-92$," At. Data Nucl. Data Tables 54(2), 181-342 (1993).

23. M. G. F. Kirsch et al., "Crab: the standard x-ray candle with all (modern) x-ray satellites," Proc. SPIE 5898, 589803 (2005).

Toru Tamagawa is a chief scientist at RIKEN Center for Pioneering Research. He is the head of the high energy astrophysics laboratory. He received his $\mathrm{PhD}$ from the University of Tokyo in 2000. He has worked for several $x$-ray satellite missions in astrophysics: HETE-2, Suzaku, MAXI, Hitomi, GEMS/PRAXyS, IXPE, and XRISM.

Biographies of the other authors are not available. 